\title{
Usefulness of a novel electrosurgical knife, the insulation-tipped diathermic knife-2, for endoscopic submucosal dissection of early gastric cancer
}

\author{
Hiroyuki Ono, Noriaki Hasuike, Tetsuya Inui, Kohei Takizana, Hisatomo Ikehara, Yuichiro Yamaguchi, \\ Yosuke Otake, and Hiroyuki Matsubayashi \\ Division of Endoscopy and GI Oncology, Shizuoka Cancer Center Hospital, 1007 Shimonagakubo, Nagaizumi-cho, Shizuoka 411-8777, Japan
}

\begin{abstract}
Background. Although endoscopic submucosal dissection (ESD) of early gastric cancer using an insulation-tipped diathermic (IT) knife enables the removal of large and ulcerative lesions en bloc, expert endoscopic skill is required. We developed an improved IT knife (IT-2) and compared its efficacy and safety with that of the original IT knife (IT-OM).

Methods. We performed ESD of 602 gastric cancers. Of these, 314 previously untreated single lesions of initial onset were analyzed. Operating time, rate of en-bloc resection, and incidence of complications were compared in the IT-2 group (161 patients) and IT-OM group (153 patients). Lesions were further analyzed as to whether they met the Japanese Gastric Cancer Association indications for ESD or extended indications.

Results. Mean resection time was significantly shorter in the IT-2 than in the IT-OM group (48 vs $63 \mathrm{~min}$ ). There were fewer surgeries lasting longer than $2 \mathrm{~h}$ in the IT-2 group than in the IT-OM group (3\% vs 12\%). En-bloc and margin-free resection rates in the IT-OM and IT-2 groups were $95 \%$ and 99\%, respectively. Perforations occurred in $3.9 \%$ of patients in the IT-OM group and in 5\% of patients in the IT-2 group (difference not significant [NS]). The incidence of postoperative hemorrhage was $7.8 \%$ in the IT-OM group and $8.7 \%$ in the IT-2 group (NS). In both groups, complications were treated endoscopically, and emergency surgery was unnecessary.

Conclusion. Resectability and complication rates were similar in the two groups. However, operating time was shorter with IT-2, irrespective of the indications for the performance of ESD. This study suggests benefits of the IT-2 over the IT-OM.
\end{abstract}

Key words IT knife $2 \cdot$ ESD $\cdot$ Gastric cancer

\section{Introduction}

Endoscopic treatment, particularly endoscopic mucosal resection (EMR) for early gastric cancer (EGC), is frequently performed in Japan. This is a result of the increase in detection of early cancers, due to physical examinations available throughout Japan, as well as advances in endoscopic instruments from the earlier gastrocameras to the newer electronic scopes. EMR is clearly superior to surgical gastrectomy and laparotomy for EGC in terms of the patient's quality of life.

Recently, the endoscopic treatment of gastric cancer has made great technical advances because of the introduction of a new procedure, endoscopic submucosal dissection (ESD). ESD has made it possible to remove large lesions exceeding $2 \mathrm{~cm}$, including those with ulcer scars, en bloc, leading to the rapid widespread use of this procedure.

An insulation-tipped diathermic knife (IT knife) was developed as an endoscopic cutting instrument for ESD at the Endoscopy Division of the National Cancer Center in Tokyo [1-3]. An insulated small ceramic sphere connected to the tip of a high-frequency needle knife allows for safe and easy incision and separation of the mucosal and submucosal layers. However, the IT knife has been recognized to have three technical drawbacks: (1) it is not easy to cut a lesion while one is looking downward, (2) the lateral cutting performance is relatively poor, and (3) because the ceramic tip is made of insulating material, it may get stuck to the cutting edge of the mucosa.

To address these issues, we developed an improved IT knife, which we call the IT knife-2 (IT-2), and here we report the results of our evaluation of its efficacy and safety in comparison with that of the original IT knife (IT-OM). 


\section{Patients and methods}

\section{IT knife-2}

In collaboration with Olympus Medical Systems (Tokyo, Japan), we planned to improve the IT-OM. To obtain good cutting ability, we made some prototypes by attaching short blades or small disks of various patterns to the long blade of the IT-OM, and we evaluated them using a porcine model. Finally, we developed the IT-2 by attaching a short three-pronged blade (Fig. 1A) perpendicular to the blade of the IT-OM (Fig. 1B).

\section{ESD indications for early gastric cancer (EGC)}

Standard indications. The standard indications for the performance of ESD proposed by the Japanese Gastric Cancer Association include: (1) differentiated adenocarcinoma, (2) intramucosal cancer, (3) lesion $20 \mathrm{~mm}$ or less in size, and (4) no ulceration [4]. Lesions that meet all of these indications are considered to present a negligible risk of lymph node metastasis [5].

Extended Indications. We extended these indications for ESD as follows: (1) differentiated adenocarcinoma, (2) intramucosal cancer, and (3) lesion regardless of size but with no ulceration or a lesion $30 \mathrm{~mm}$ or less with ulceration. Lesions meeting these criteria have been shown by us to present a negligible risk of lymph node metastasis [5].

\section{Patients}

We performed ESD for a total of 602 gastric cancer lesions, from September 2002 to December 2005, at the Shizuoka Cancer Center. Of these lesions, 169 were synchronous multiple lesions, 34 were recurrent cancers after endoscopic treatment, 4 were cancers in the remnant stomach, 67 did not meet indications for ESD, and 14 were asynchronous second or third cancers. For the present study, we excluded these lesions. In brief, we retrospectively analyzed 314 previously untreated single lesions of initial onset that met the indications for ESD. Endoscopists selected either the IT-2 or IT-OM according to their individual preference. Of the 314 subjects, 153 were treated with the IT-OM (IT-OM group) and 161 with the IT-2 (IT-2 group). Table 1 shows patient characteristics in each group. There were no significant differences between groups in age, sex, ulcer findings, tumor size, or size of the resected specimen. However, there were differences in tumor location between the two groups. The upper third: middle third: lower third ratio was $31: 52: 70$ in the IT-OM group and $31: 77: 53$ in the IT-2 group $(P=0.030)$. The ratio of patients receiving treatment according to the standard indications or extended indications also differed significantly between the groups $(P=0.027)$. In the IT-OM group, 105 patients were treated under standard indications and 48 under extended indications, while in the IT-2 group, the standard indications were applied in 90 and extended indications in 71 patients. Informed consent was obtained from all patients in written form.

Table 1. Patient characteristics

\begin{tabular}{|c|c|c|c|}
\hline & Original IT knife & IT knife-2 & \\
\hline Number of patients & 153 & 161 & NS \\
\hline Age, years median (range) & $68(37-91)$ & $69(39-90)$ & NS \\
\hline Sex F:M & $48: 105$ & $37: 124$ & NS \\
\hline Ulcer findings positive:negative & $30: 123$ & $37: 124$ & NS \\
\hline Tumor size, mm mean (range) & $23(2-84)$ & $26(3-110)$ & NS \\
\hline Size of resected specimen (mm) & $48(16-105)$ & $51(13-120)$ & NS \\
\hline Location of cancer Upper:middle:lower & $31: 52: 70$ & $31: 77: 53$ & $P=0.030$ \\
\hline Indication Standard: extended & $105: 48$ & $90: 71$ & $P=0.027$ \\
\hline
\end{tabular}

IT knife, insulation-tipped diathermic knife
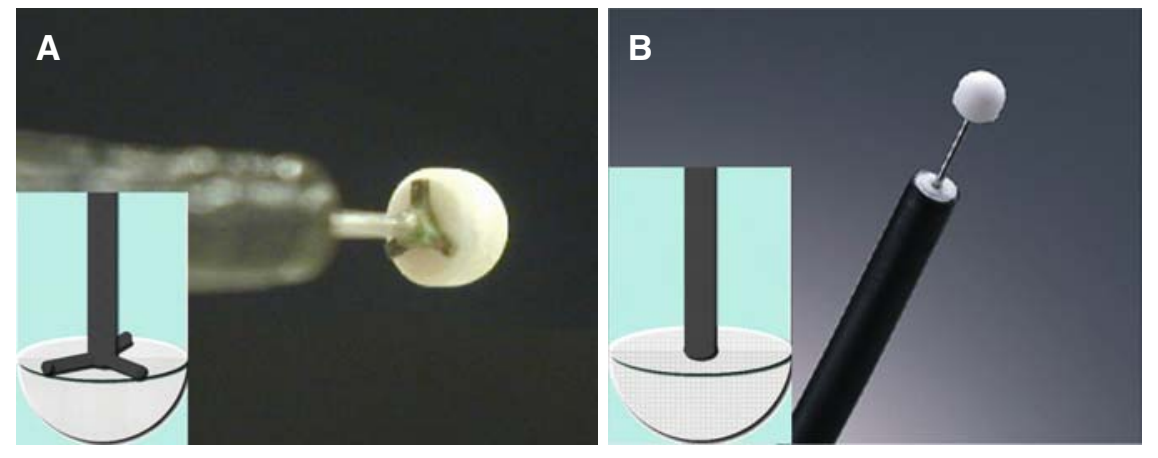

Fig. 1A, B. Images of the original model insulation-tipped diathermic knife (ITOM) (B) and the new IT knife-2 (A). The IT knife-2 has an insulation tip with three blades extending to the edge of the tip, creating superior sharpness 


\section{Parameters analyzed}

The en-bloc resection rate, operating time, and incidence of complications (perforation and postoperative hemorrhage) were compared in the two patient groups. Complete resection was defined as en-bloc resection with pathological negativity for cancer cells at the lateral and perpendicular stumps. Operating time was measured from the time of circumferential marking to the time of the completion of resection. Postoperative hemorrhage was defined as positive when postoperative endoscopic hemostasis was needed. To decrease the bias contingent on operator skill, three endoscopists were selected who had performed more than 150 ESDs using the IT-OM. Because this study was not a randomized controlled trial, we performed exploratory research to compare results between the IT-2 and IT-OM groups.

\section{ESD technique}

We used essentially the same technique with both the IT-2 and IT-OM. At 3 to $5 \mathrm{~mm}$ away from the peripheral border of the lesion, the entire circumference was marked using a needle knife (September 2002 to June 2005) or an argon plasma coagulation probe (July 2005 to December 2005), after which normal saline (September 2002 to June 2005) or 10\% glycerin solution (July 2005 to December 2005) was injected into the submucosal layer. An incision was then made with the needle knife to the muscularis mucosae. Once the ceramic ball at the tip of the IT knife was inserted, an incision was made around the entire circumference. Subsequently, the submucosal layer was resected (Fig. 2). After the completion of resection, a nasogastric tube was positioned to monitor postoperative hemorrhage overnight.

\section{Statistical analysis}

Data were analyzed using Fisher's exact probability test and Student's $t$-test. A $P$ value of less than 0.05 was considered significant.

\section{Results}

\section{Operating time}

The mean operating time for resection was significantly shorter in the IT-2 group (mean, $48 \mathrm{~min}$; range, 7 to $300 \mathrm{~min}$; median, $35 \mathrm{~min}$ ) than in the IT-OM group (mean, $63 \mathrm{~min}$; range, 15 to $390 \mathrm{~min}$; median, $40 \mathrm{~min}$; Table 2). When the mean operating time was analyzed according to indications, it was significantly shorter in the IT-2 group than in the IT-OM group for those with both standard and extended indications. Further, significantly fewer patients in the IT-2 group than in the IT-OM group underwent a procedure that took longer than $2 \mathrm{~h}$ (Table 2).

\section{Rate of en-bloc and margin-free resection}

The rate of en-bloc and margin-free resection for the IT-OM group was $95 \%$, and that for the IT-2 group was $99 \%$; both were satisfactory results (Table 3). When analyzed according to the difference in indications, similar rates for the IT-OM group (96\% and 94\% for standard and extended indications, respectively) and for the IT-2 group ( $100 \%$ and $99 \%$, respectively) were obtained irrespective of whether standard or extended indications were applied.

\section{Complications}

Perforations occurred in $3.9 \%$ of IT-OM patients and in $5 \%$ of IT-2 patients, with no significant difference between the two groups (Table 4). The incidence of complications, which included perforation and postoperative hemorrhage, did not differ significantly between the two groups, irrespective of the difference in indications. In both groups, complications were managed endoscopically, and patients were discharged within 7 days after endoscopic treatment. Emergency surgery was not necessary in any patient.

\section{Discussion}

The obstacles associated with ESD in general are that it is time-consuming and technically difficult, and complications such as perforation and hemorrhage sometimes cannot be avoided. Following the introduction of the IT-OM, many other devices have been reported for ESD [6-8]. While these devices were shown to have both advantages and disadvantages, the IT-OM remained superior in terms of operating time. However, as mentioned in the Introduction, the IT-OM is associated with some drawbacks.

In the present study, we demonstrated that the IT-2, a modified form of the IT-OM, had advantages in operability compared with the IT-OM, based on the significantly shorter operating time in the IT-2 group than in the IT-OM group. This was despite the fact that there were more patients with extended indications in the IT- 2 group than in the IT-OM group and despite the fact that the lesion was more frequently in a difficult location (corpus lesion) in the IT-2 group. Also, the rates of en-bloc and margin-free resection were almost the same in both groups. The reason for the higher number of difficult cases in the IT-2 group compared to 

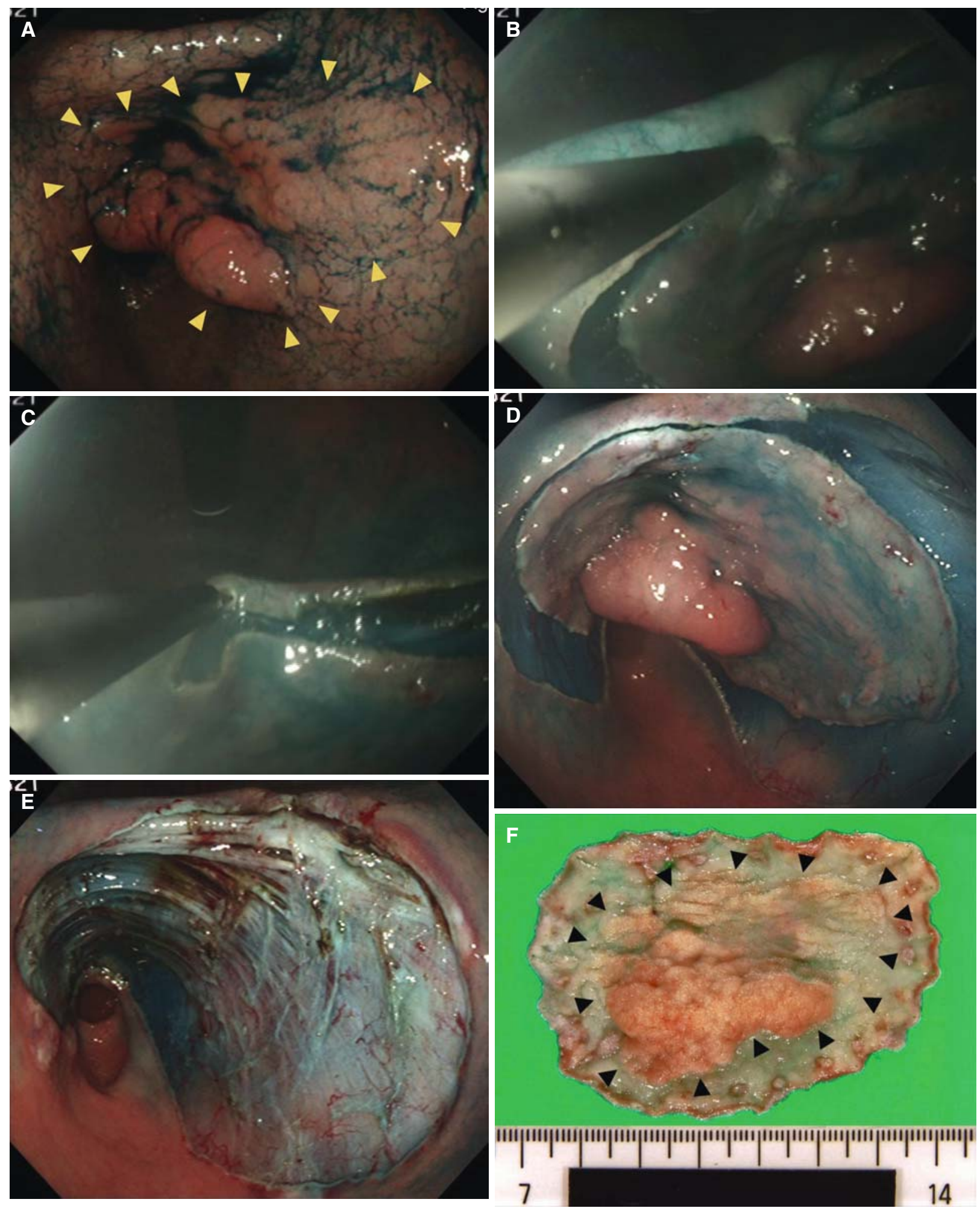

Fig. 2A-F. Actual endoscopic submucosal dissection (ESD), using the IT knife-2. A cancerous lesion, an early gastric cancer, macroscopic type $0 \mathrm{I}+\mathrm{IIa}$, and $48 \mathrm{~mm}$ in diameter, is located on the lesser curvature up to the posterior wall of the gastric antrum. Yellow arrowheads show the margin of the lesion (A). Lateral cutting is difficult to perform in such a position, but with the IT knife-2, cutting can be performed

beautifully. It is far easier to cut laterally with the IT knife-2 than with the original model IT knife $(\mathbf{B}, \mathbf{C}, \mathbf{D}, \mathbf{E})$. The lesion was removed en bloc and was confirmed as intramucosal cancer histologically. Black arrowheads show the margin of the lesion (F). Operation time (from beginning of marking to retrieving the lesion) was $22 \mathrm{~min}$ 
Table 2. Comparison of operation times between groups in which the old model IT knife and the new IT knife-2 were used

\begin{tabular}{|c|c|c|c|}
\hline & $\begin{array}{c}\text { Original IT knife } \\
\quad n=153\end{array}$ & $\begin{array}{c}\text { IT knife- } 2 \\
n=161\end{array}$ & \\
\hline \multicolumn{4}{|l|}{ Overall, min } \\
\hline Mean (range) & $63(15-390)$ & $48(7-300)$ & $P=0.015$ \\
\hline Median & 40 & 35 & \\
\hline Within $60 \min (\%)$ & $75 \%(114 / 153)$ & $75 \%(120 / 161)$ & NS \\
\hline Over $120 \min (\%)$ & $12 \%(18 / 153)$ & $3 \%(5 / 161)$ & $P=0.008$ \\
\hline Standard indication $(n=195)$ & $n=105$ & $n=90$ & \\
\hline Mean (range) & $47(15-320)$ & $32(7-110)$ & $P=0.003$ \\
\hline Median & 35 & 25 & \\
\hline Within $60 \min (\%)$ & $85 \%(89 / 105)$ & $90 \%(81 / 90)$ & NS \\
\hline Over $120 \min (\%)$ & $6 \%(6 / 105)$ & $0 \%(0 / 90)$ & $P=0.034$ \\
\hline Extended indication $(n=119)$ & $n=48$ & $n=71$ & \\
\hline Mean (range) & $96(15-390)$ & $68(16-300)$ & $P=0.026$ \\
\hline Median & 60 & 60 & \\
\hline Within $60 \min (\%)$ & $52 \%(25 / 48)$ & $59 \%(42 / 71)$ & NS \\
\hline Over $120 \min (\%)$ & $25 \%(12 / 48)$ & $7 \%(5 / 71)$ & $P=0.008$ \\
\hline
\end{tabular}

Table 3. Comparison of the rates of en-bloc and margin-free resection between groups in which the old model IT knife and the new IT knife-2 were used

\begin{tabular}{llll}
\hline & $\begin{array}{c}\text { Original IT knife } \\
n=153\end{array}$ & \multicolumn{1}{c}{$\begin{array}{c}\text { IT knife-2 } \\
n=161\end{array}$} & \\
\hline En-bloc and margin-free resection, $\%$ & $95 \%(146 / 153)$ & $99 \%(160 / 161)$ & NS \\
Standard indication $(n=195)$ & $n=105$ & $n=90$ & NS \\
& $96 \%(101 / 105)$ & $100 \%(90 / 90)$ & NS \\
Extended indication $(n=119)$ & $n=48$ & $n=71$ & \\
& $94 \%(45 / 48)$ & $99 \%(70 / 71)$ & \\
\hline
\end{tabular}

Table 4. Comparison of complications between groups in which the old model IT knife and the new IT knife-2 were used

\begin{tabular}{lcll}
\hline & Original IT knife & \multicolumn{1}{c}{ IT knife-2 } \\
& \multicolumn{1}{c}{$n=153$} & \multicolumn{1}{c}{$n=161$} & \\
\hline Perforation & $3.9 \%(6 / 153)$ & $5.0 \%(8 / 161)$ & NS \\
Standard indication $(n=195)$ & $n=105$ & $n=90$ & NS \\
& $3.8 \%(4 / 105)$ & $3.3 \%(3 / 90)$ & NS \\
Extended indication $(n=119)$ & $n=48$ & $n=71$ & \\
& $4.2 \%(2 / 48)$ & $7.0 \%(5 / 71)$ & NS \\
Postoperative hemorrhage & $7.8 \%(12 / 153)$ & $8.7 \%(14 / 161)$ & NS \\
Standard indication $(n=195)$ & $n=105$ & $n=90$ & NS \\
& $6.7 \%(7 / 105)$ & $8.9 \%(8 / 90)$ & $n=71$ \\
Extended indication $(n=119)$ & $n=48$ & $8.5 \%(6 / 71)$ & \\
& $10 \%(5 / 48)$ & & \\
\hline
\end{tabular}

the IT-OM group may be that experienced endoscopists perceived the superiority of the IT-2 over the IT-OM in cutting capability and selected it for difficult lesions. The advantage of the IT-2 over the IT-OM was even more evident when we compared the number of cases that required an operating time of longer than $2 \mathrm{~h}$; such cases were significantly fewer in the IT-2 than in the IT-OM group, regardless of whether standard or extended indications were applied.
The most plausible explanation for this advantage of the IT- 2 is that its cutting ability, including lateral cutting capability, and cutting speed were superior to those of the IT-OM. It is speculated that the reason for this good cutting capability is that the three short blades attached to the ceramic tip allow the catching of mucosal and submucosal tissue more securely than the IT-OM, whose ceramic tip (which is without short blades) may get stuck. With regard to the safety issue, the rates of per- 
foration and postoperative hemorrhage did not differ between the two groups, although the IT- 2 group consisted of more complicated cases. However, these rates of complications were apparently higher than those reported with conventional EMR, such as the strip biopsy method $[9,10]$. Nevertheless, perforations made by IT knives can usually be repaired endoscopically with endoclips (Olympus Medical Systems, Tokyo, Japan) [11], and we found, in the present study, that all the patients with postoperative hemorrhage could be conservatively treated, except for one patient who refused a nasogastric tube and for whom blood transfusion was necessary. Incidentally, it was considered that the high incidence of postoperative hemorrhage with the use of the IT knives might have occurred because we inserted a nasogastric tube in each patient after ESD to monitor and detect postoperative hemorrhage earlier than would usually be possible without this maneuver. Because this study was not a randomized control trial, we cannot deny selection bias; it would be necessary to carry out a randomized control trial to achieve scientific truth. However, considering our finding of the advantage of the IT- 2 over the IT-OM in adverse conditions (that is, the IT-2 group included more difficult lesions compared with the IT-OM group), we think our good evaluation of the IT- 2 is reasonable.

In summary, the results of the present study clearly indicate the superiority of the IT- 2 over the IT-OM, particularly in shortening operating time.

Acknowledgments The authors thank Professor Yoshiro Niitsu, Fourth Department of Internal Medicine, Sapporo Medical University, for his valuable advice and English-language editing. This work was supported in part by a Grant-in-Aid from the Ministry of Health, Labour, and Welfare of Japan.

\section{References}

1. Ono H, Gotoda T, Kondo H, Yamaguchi H, Kozu T, Fujii $T$, et al. A new method of EMR using insulation-tipped diathermia knife (in Japanese, with English abstract). Endosc Dig 1999;11: 675-81.

2. Hosokawa K, Yoshida S. Recent advances in endoscopic mucosal resection for early gastric cancer (in Japanese, with English abstract). Jpn J Cancer Chemother 1998;25:476-83.

3. Ono H, Kondo H, Gotoda T, Shirao K, Yamaguchi H, Saito D, et al. Endoscopic mucosal resection for treatment of early gastric cancer. Gut 2001;48:225-9.

4. Japanese Gastric Cancer Association. Gastric cancer treatment guideline (in Japanese). Tokyo: Kanehara; 2004.

5. Gotoda T, Yanagisawa A, Sasako M, Ono H, Nakanishi Y, Shimoda T, et al., Incidence of lymph node metastasis from early gastric cancer: estimation with a large number of cases at two large centers. Gastric Cancer 2000;3:219-25.

6. Oyama T, Kikuchi Y. Aggressive endoscopic mucosal resection in the upper GI tract: hook knife EMR method. Minim Invasive Ther Allied Technol 2002;11:291-95.

7. Yahagi N, Fujishiro M, Kakushima N, Kobayashi K, Hashimoto T, Oka M, et al. Endoscopic submucosal dissection for early gastric cancer using the tip of an electro-surgical snare (thin type). Dig Endosc 2004;16:34-8.

8. Yamamoto H, Sekine Y, Higasizawa T, Kihara K, Kaneko Y, Hosoya Y, et al. Successful en bloc resection of a large superficial gastric cancer by using sodium hyaluronate and electrocautery incision forceps. Gastrointest Endosc 2001;54:629-33.

9. Kojima T, Parra-Blanco A, Takahashi H, Fujita R. Outcome of endoscopic mucosal resection for early gastric cancer: review of the Japanese literature. Gastrointest Endosc 1998;48:550-5.

10. Kaneko E, Hanada H, Kasugai T, Ogoshi K, Niwa K. The survey of gastrointestinal endoscopic complications in Japan (in Japanese). Gastroenterol Endosc 2000;42:308-13.

11. Minami S, Gotoda T, Ono H, Oda I, Hamanaka H. Complete endoscopic closure of gastric perforation induced by endoscopic resection of early gastric cancer using endoclips can prevent surgery. Gastrointest Endosc 2006;63:596-601. 